\title{
TAC Zoo: Uma Adaptação Digital do Teste de Atenção por Cancelamento para o TDAH
}

\author{
Bruno C. Matias, Victor T. Sarinho \\ ${ }^{1}$ Laboratório de Entretenimento Digital Aplicado - LEnDA \\ Universidade Estadual de Feira de Santana - UEFS \\ Feira de Santana - Bahia - Brasil \\ brunoclaudinomatias@gmail.com, vsarinhoduefs.br
}

\begin{abstract}
The effects of Attention Deficit Hyperactivity Disorder (ADHD) begin in childhood and can continue throughout life. People affected by this disorder tend to have greater difficulties in routine activities when compared to people who do not have the disorder. This article presents the construction of a digital game that seeks to adapt the Cancellation Attention Test applied to the diagnosis of ADHD. As a result, a gamified tool aimed at children was obtained, capable of collecting metrics to support the diagnosis of ADHD in a simple and automated way.
\end{abstract}

Resumo. Os efeitos do Transtorno de Déficit de Atenção e Hiperativade (TDAH), começam na infância e podem continuar durante toda a vida. As pessoas afetadas por esse transtorno tendem a apresentar maiores dificuldades em atividades rotineiras quando comparada a pessoas que não possuem o transtorno. Este artigo apresenta a construção de um jogo digital que busca adaptar o Teste de Atenção por Cancelamento aplicado no diagnóstico de TDAH. Como resultado, obteve-se um jogo que pode ser usado como ferramenta gamificada voltada para o público infantil capaz de coletar métricas de apoio ao diagnóstico de TDAH de uma maneira simples e automatizada.

\section{Introdução}

O Transtorno de Déficit de Atenção e Hiperatividade (TDAH) tem como principais características a desatenção, a hiperatividade e a impulsividade [Catelan-Mainardes 2010], afetando o indivíduo não apenas socialmente, mas também no seu desempenho acadêmico e profissional [Rohde et al. 2004]. Trata-se de um transtorno com diagnóstico crescente e é um dos distúrbios neuropsiquiátricos mais comuns na infância.

O TDAH se desenvolve geralmente antes dos sete anos de idade, e é diagnosticado com mais frequência no período escolar da criança, principalmente quando as dificuldades de atenção, concentração e inquietude são percebidas quando comparadas com outras crianças da mesma idade [Couto et al. 2010]. Como resultado, o TDAH representa, junto com a dislexia, a principal causa de fracasso escolar de crianças e adolescentes em geral [Couto et al. 2010].

Apesar de não existir uma bateria fixa de testes para diagnosticar as crianças com TDAH [Hazin et al. 2012], existem propostas mais simples de avaliação que aplicam testes já existentes. Um exemplo é o Teste de Atenção por Cancelamento (TAC), cujo objetivo da pessoa que está sendo testada é assinalar as imagens que forem iguais a figura 
alvo, a qual é composta de níveis contendo matrizes de 6 figuras geométricas diferentes, de cor preta e de fundo branco [Parisod et al. 2014].

Jogos digitais são uma das principais formas de acesso a tecnologia para crianças [Gros 2003]. Eles também são definidos como "ambientes atraentes e interativos que capturam a atenção do jogador ao oferecer desafios que exigem níveis crescentes de destreza e habilidades" [Savi and Ulbricht 2008]. Dentro do contexto de jogos digitais existem os jogos sérios ou serious games, que, diferente dos jogos normais que só possuem o intuito de divertir, possuem o propósito principal de transmitir o conhecimento [Michael and Chen 2005].

Jogos digitais podem se tornar uma ótima maneira de melhorar o contato com indivíduos, tendo como resultado um espaço significativo para a ampliação dos mesmo no âmbito da reabilitação de jovens e adultos em geral [Rocha et al. 2014]. Jogos também podem atuar como ferramenta de apoio ao tratamento e suporte de pessoas portadoras do TDAH [Maciel et al. 2019], tendo inclusive o apoio de profissionais da área se mostrando otimistas sobre isso. Contudo, também é possível observar que a maioria dos jogos voltados para TDAH são voltados ao tratamento em si, enquanto que os trabalhos voltados a conscientização e ao diagnóstico em si são escassos [Maciel et al. 2019].

Assim, pensando no uso de jogos digitais como ferramenta para atrair a atenção de crianças e adolescentes, desenvolveu-se o TAC Zoo, um jogo que usa elementos de gamificação para prender a atenção e deixar o Teste de Atenção por Cancelamento (TAC) mais atrativo e dinâmico para o público infantil. Nele, os elementos monocromáticos do teste original tomam vida através de animais coloridos em um ambiente digital interativo, nos quais o jogador deve marcar o máximo de animais corretos antes que o seu tempo acabe.

\section{Teste de Atenção por Cancelamento (TAC)}

O TAC é composto por três matrizes compostas por 6 figuras geométricas (traço, cruz, quadrado, círculo, estrela e triângulo) de cor preta, impressas em um fundo branco. Essas figuras geométricas são os estímulos, e o usuário deve assinalar todos os estímulos que são iguais aos estímulos alvos que foram mostrados anteriormente. Ou seja, haverá um estímulo específico que deve ser encontrado o máximo de vezes possível na matriz.

O primeiro nível do teste (Figura 1) é destinado à avaliação da atenção seletiva, medindo a capacidade do indivíduo de diferenciar um estímulo específico em meio aos outros. Nesse nível, a matriz de estímulos contém 18 linhas, com cada linha sendo composta de 20 figuras. Em cada quadrante, cada estímulo (ou figura) aparece 15 vezes. Ou seja, se dividirmos a matriz em quatro partes, cada uma terá 15 vezes o primeiro estímulo, 15 vezes o segundo estímulo, 15 vezes o terceiro, e assim por diante.

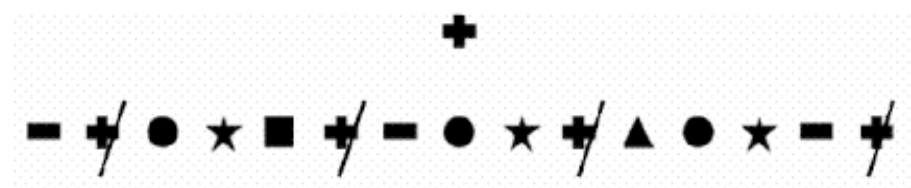

Figura 1. Estímulo alvo e primeira linha da primeira matriz do TAC.

O segundo nível (Figura 2) tem o mesmo objetivo do primeiro, mas com uma 
dificuldade maior. A matriz tem o mesmo tamanho, mas agora existem dois estímulos alvos que devem ser achados na matriz. Além disso, os alvos só aparecem 12 vezes na matriz, sendo 3 por quadrante. O terceiro e último nível (Figura 3) é usado para testar a capacidade do indivíduo de mudar o estímulo alvo, ou seja, mudar o foco durante o processo. Para isso, no lugar de um ou dois estímulos alvos acima, agora toda linha terá um alvo diferente. Além disso, para cada quadrante, um estímulo se repetirá 15 vezes, mas o estímulo alvo daquela linha deverá se repetir de 2 a 6 vezes.

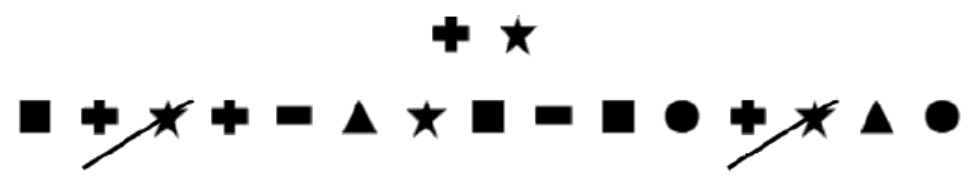

Figura 2. Estímulos alvos e primeira linha da segunda matriz do TAC.

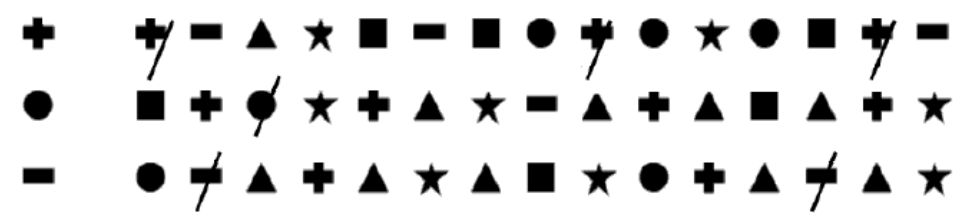

Figura 3. Estímulos alvos e as três primeiras linhas da terceira matriz do TAC.

Em todos os níveis o tempo para que o indivíduo marque os estímulos é de um minuto. A partir do que for marcado pelo jogador, três escores são computados: estímulos corretos marcados, estímulos errados marcados, e estímulos corretos não marcados.

\section{Jogo Produzido}

Conforme o nome proposto para o jogo, TAC Zoo apresenta uma tela inicial representando a entrada de um zoológico com diferentes animais observando uma criança que irá entrar nessa "aventura" (Figura 4). Para o menu inicial, este é representado por diferentes placas de direções, indicando opções de configurações de som e linguagem, de informações sobre o jogo e de sair do mesmo. Vale salientar que os elementos do menu inicial e os animais do jogo que são usados como estímulo foram desenhados em sua maioria com cores no eixo azul-amarelo, tendo como base a premissa de que portadores de TDAH teriam mais dificuldade de prestar atenção nessa configuração [Maciel et al. 2019].

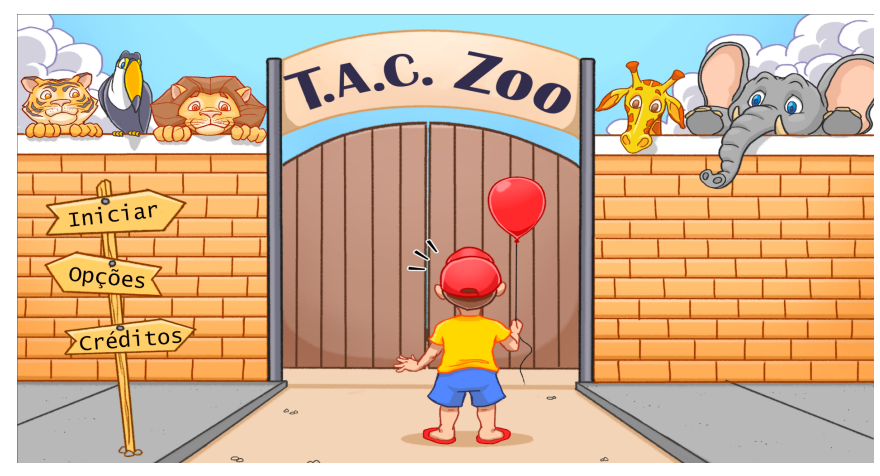

Figura 4. Menu principal do TAC Zoo. 
No primeiro nível do jogo (Figura 5), é possível verificar o estímulo alvo que se deve buscar e a matriz com os 360 estímulos (18 linhas e 20 colunas) a serem avaliados. Quando um dos animais (estímulos) é selecionado, o algoritmo verifica se ele bate com o estímulo alvo. Se for o correto, em cima da figura é assinalado como correto (clicando na figura com o mouse do desktop ou notebook), caso contrário é assinalado com um símbolo em vermelho de errado (Figura 5). Após ter selecionado um dos estímulos, não é possível apagar a marca, já que o valor de estímulos errados marcados são usados para gerar um escore final para o jogador.

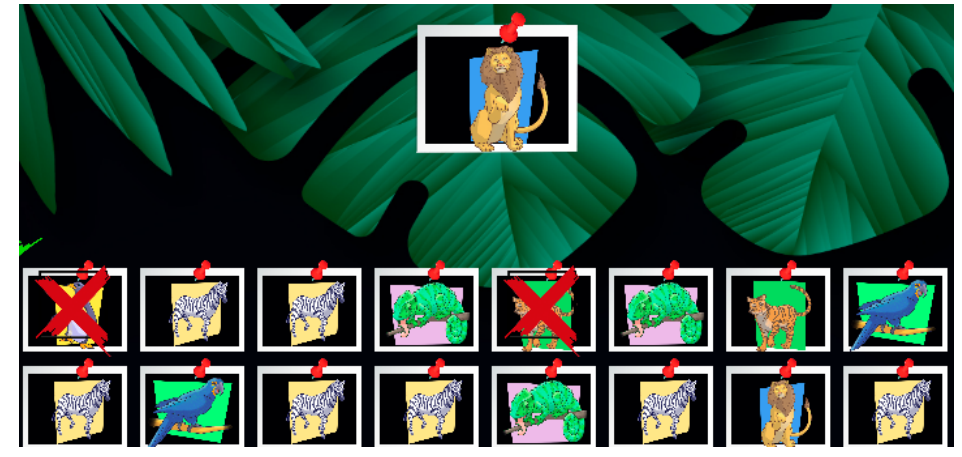

Figura 5. Tela de jogo do TAC Zoo com zoom no estímulo e na primeira linha.

Ao entrar em um nível, o jogo fica pausado. O fundo fica desfocado e apresenta um botão de começar no centro da tela. Quando pressionado, o jogo é ativado e o tempo começa a contar. No canto superior esquerdo é mostrado o tempo que falta para o nível acabar, e, depois que o cronômetro chegar a zero, os animais não podem ser selecionados e uma mensagem de tempo esgotado aparece (Figura 6), assim como o botão para continuar e carregar o próximo nível.

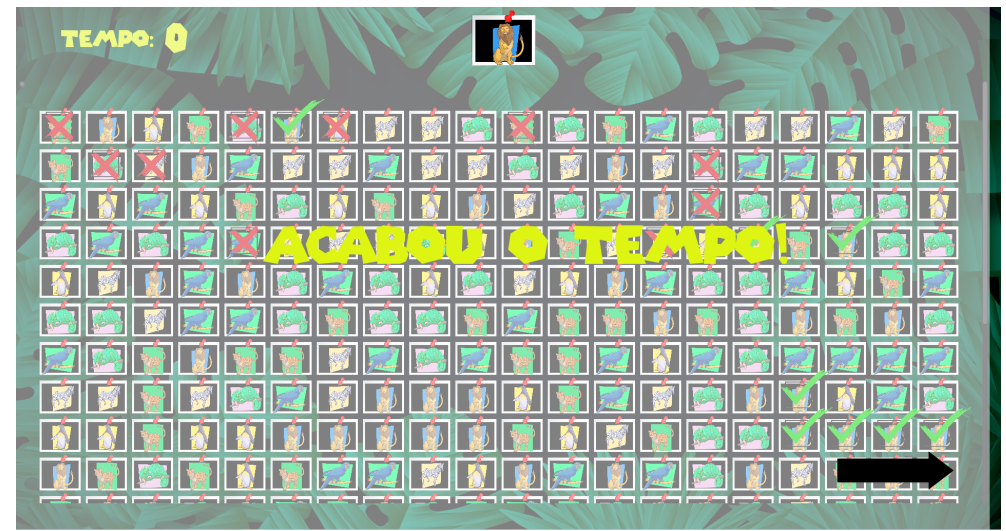

Figura 6. Tela de tempo esgotado do nível no jogo TAC Zoo.

Os demais níveis do jogo seguem funcionando da mesma forma que as regras originais do TAC, porém apresentam as variações estéticas e de mecânicas digitais propostas para o TAC Zoo (Figura 7).

Como resultados obtidos com a execução do jogo, três escores (acertos, erros e estímulos esquecidos) são salvos em um banco de dados interno, permitindo que os resultados do jogador sejam comparado com os outros dados pré-existentes. Assim, o profissional de saúde responsável poderá ter acesso não somente a pontuação do indivíduo 


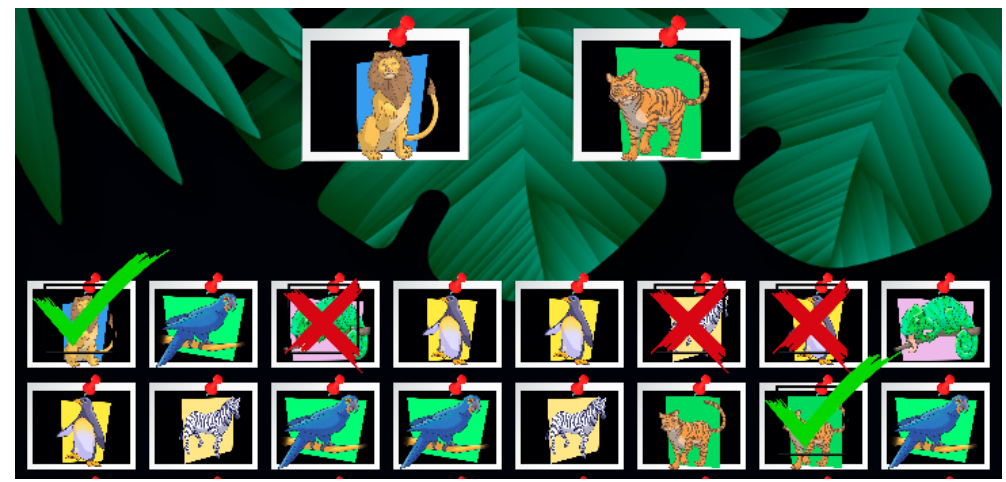

Figura 7. Segundo nível do TAC Zoo com zoom no topo e dois estímulos a serem selecionados pelo jogador.

testado, mas também terá uma análise estatística dos dados do indivíduo em relação a outros que possuam mesma faixa etária, para que haja uma melhor comparação para auxiliar no seu diagnóstico final.

A construção do jogo foi feita com auxílio da ferramenta Godot 3, um motor de jogos de código aberto que possuí uma grande gama de recursos para a construção de jogos [Godot 2019]. A aplicação atualmente é exportado para ser executados em computadores (desktop ou notebook). E o jogo foi feito para que a pessoa a ser testada possa usar somente cliques do mouse para realizar o teste sem outras preocupações de comandos.

\section{Conclusões e Trabalhos Futuros}

Jogos possuem um grande impacto no tratamento de pessoas portadoras do TDAH, porém ainda existe uma grande demanda por ferramentas digitais que possam auxiliar no diagnóstico deste transtorno [Maciel et al. 2019]. Neste sentido, o TAC Zoo representa uma adaptação do TAC para o diagnóstico de TDAH, no sentido de criar um jogo digital, com mecânicas simples conforme o TAC, porém dentro de uma temática infantil de zoológico inicialmente proposta.

Com relação a adaptação realizada no TAC pelo TAC Zoo, tem-se que no TAC original os elementos são representados por figuras simples, o que possibilita usar uma grande quantidade de objetos juntos na matriz proposta. Já no TAC Zoo, este segue o modelo proposto no teste original, porém com figuras mais complexas, gerando como resultado uma quantidade excessiva de informação visual para o jogador. Neste sentido, apesar do TAC Zoo apresentar um ambiente mais atrativo para crianças, se faz necessário avaliar até que ponto o tamanho da matriz impacta no desempenho dos jogadores e na validade do TAC aplicado, quando se utiliza de imagens com uma maior quantidade de informações visuais para o jogador.

Como trabalhos futuros, pretende-se produzir uma versão onde as imagens podem ser configuradas pelo jogador, permitindo ampliar assim as possíveis temáticas, sendo estas infantis ou não, a serem aplicadas no TAC, bem como gerar versões de imagens customizadas dentro do contexto da vida cotidiano do jogador. A possibilidade de se configurar o tamanho da matriz de teste, de modo a gerar níveis de dificuldades distintos a serem aplicados, conforme a decisão do responsável pela execução do teste, também será realizada em um futuro próximo. E para finalizar, será realizado a coleta de dados 
on-line de partidas realizadas, de modo a criar um banco de dados por paciente, bem como um comparativo de métricas obtidas com pacientes distintos, para fins de identificação de padrões de jogo adotados por portadores de TDAH, algo não realizado pelo TAC original que efetua apenas uma comparação dos três escores criados com faixa de valores fixos para definir o desempenho de apenas um indivíduo.

\section{Agradecimentos}

Agradecimentos ao Programa de Bolsa de Iniciação científica da Universidade Estadual de Feira de Santana (PROBIC/UEFS) pela concessão da Bolsa de Pesquisa para este projeto.

\section{Referências}

Catelan-Mainardes, S. C. (2010). Transtorno de déficit de atenção e hiperatividade na infância e adolescência pela perspectiva da neurobiologia. Saúde e Pesquisa, 3(3).

Couto, T. S., Melo-Junior, M. R., and Gomes, C. R. A. (2010). Aspectos neurobiológicos do transtorno do déficit de atenção e hiperatividade (tdah): uma revisão. Ciências \& Cognição, 15(1):pp-241.

Godot (2019). The game engine you waited for. https://godotengine.org/.

Gros, B. (2003). The impact of digital games in education. First Monday, 8(7):6-26.

Hazin, I., da Rocha Falcão, J. T., Garcia, D., Gomes, E., Cortez, R., Maranhão, S., Menezes, T., and Dias, M. d. G. B. B. (2012). Dados normativos do teste de atenção por cancelamento (tac) em estudantes do ensino fundamental. Psico, 43(4):1.

Maciel, V. D., Matias, B. C., and Sarinho, V. T. (2019). Uma revisão sistemática da literatura de jogos digitais voltados para o tdah. In Anais do XIX Simpósio Brasileiro de Computação Aplicada à Saúde, pages 130-140. SBC.

Michael, D. R. and Chen, S. L. (2005). Serious games: Games that educate, train, and inform. Muska \& Lipman/Premier-Trade.

Parisod, H., Pakarinen, A., Kauhanen, L., Aromaa, M., Leppänen, V., Liukkonen, T. N., Smed, J., and Salanterä, S. (2014). Promoting children's health with digital games: A review of reviews. Games for Health: Research, Development, and Clinical Applications, 3(3):145-156.

Rocha, P., Nery Filho, J., and Alves, L. (2014). Jogos digitais e reabilitação neuropsicológica: delineando novas mídias. Anais do Seminário Tecnologias Aplicadas a Educação e Saúde, 1(1).

Rohde, L. A. P., Miguel Filho, E. C., Benetti, L. P. d. C., Gallois, C. B., and Kieling, C. C. (2004). Transtorno de déficit de atenção/hiperatividade na infância e na adolescência: considerações clínicas e terapêuticas. Revista de psiquiatria clínica. São Paulo. Vol. 31, n. 3 (2004), p. 124-131.

Savi, R. and Ulbricht, V. R. (2008). Jogos digitais educacionais: benefícios e desafios. RENOTE, 6(1). 